\title{
STATISTICAL FINITE ELEMENT ANALYSIS FOR BONE MODELING
}

\author{
Philippe Büchler (1), Nils Reimers (2), Daniel Rückert (3), Mauricio Reyes (1), Miguel \\ González (1)
}

1. MEM Research Center, University of Bern, Switzerland

2. Stryker Osteosynthesis, Kiel, Germany

3. Department of Computing, Imperial College, London, United Kingdom

\section{Introduction}

Current implant design techniques in orthopaedics make use of limited information about the characteristics of the target bone. Evaluation and optimization of the implant is done by manual fitting and fixation procedures, applied on a small set of cadaver bones in a trial-and-error process to find the optimal shape and mechanical properties. Therefore, it is difficult to assess whether the set of implant shapes will fit most of the population.

In the present study, we propose a method to represent the distribution of bone shape of a given population. The model is based on large collections of CT scans of the targeted population and statistical tools are used to define the average bone shape of the population as well as the most relevant variations of the bone shape around this averaged model. Since bone mechanical properties are related to the Hounsfield Units given by calibrated CT scans. The developed statistical model includes the distribution of local bone mechanical propertied in the population. When combined with finite element calculations, the proposed technique enables virtual implant testing over a complete population.

\section{Materials and Methods}

$\mathrm{CT}$ of the proximal femur were used for the construction of a statistical model. These scans were segmented to delimit the region of interest of the bone structure. One femur from the training set was chosen as the reference bone and the rest of the data sets were rigidly registered to the reference bone. A statistical model of shape and intensity is computed by means of principal component analysis. The model contains the average bone and the principal modes of variation and allows the generation of further valid bones instances.

In a second step finite element analysis (FE) were performed on representative instances generated with the statistical model. The bone geometries were meshed with 3D elements. Boundary conditions corresponded to a two times body weight on the femoral head. The bone mechanical properties were non homogenous. ABAQUS was used for the simulations.

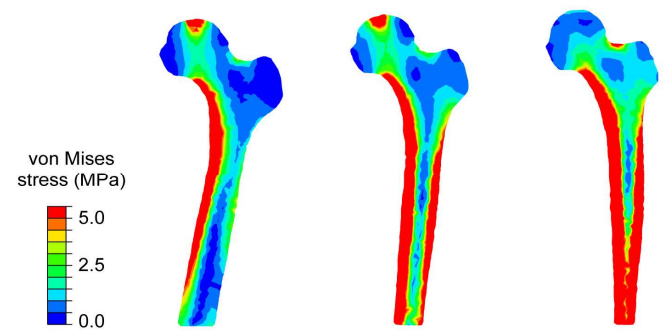

Fig.1: Stress distribution (-2sd, average and $+2 s d)$ corresponding to a load applied on the head.

\section{Discussion:}

A framework to a combined statistical model of shape and density with finite element calculations was presented. This technique has many potential applications in orthopaedic research and implant design. Virtual testing of new implants will in the future replace cadaver testing. Further, being able to study the whole range of bone shapes and densities of the target population will lead to better fitting implants, as well as a considerable cost reduction in the design process. In order to assess the qualities of an implant, further developments will enable the testing of different scenarios concerning implant position or fixation techniques.

By sampling the statistical model, which contains the variation in shape and bone density across the population, and performing the finite element experiment on each instance, we are able to reconstruct the statistical distribution of biomechanical behaviour of implants across a given population. A complementary application of these techniques will be the patient-specific pre-clinical evaluation of an implant, taking into account the particular skeletal anatomy, bone quality, and implant position to assess the biomechanical performance of the implant on the patient.

\section{Acknowledgements}

This research was funded by the Swiss National Science Foundation (NCCR-CoMe) and the Swiss Innovation Promotion Agency (CTI). 